\title{
Qualidade de vida de pacientes internados submetidos ao transplante de células tronco hematopoiéticas*
}

\section{Quality of life of hospitalized patients submitted to hematopoietic stem cells transplantation}

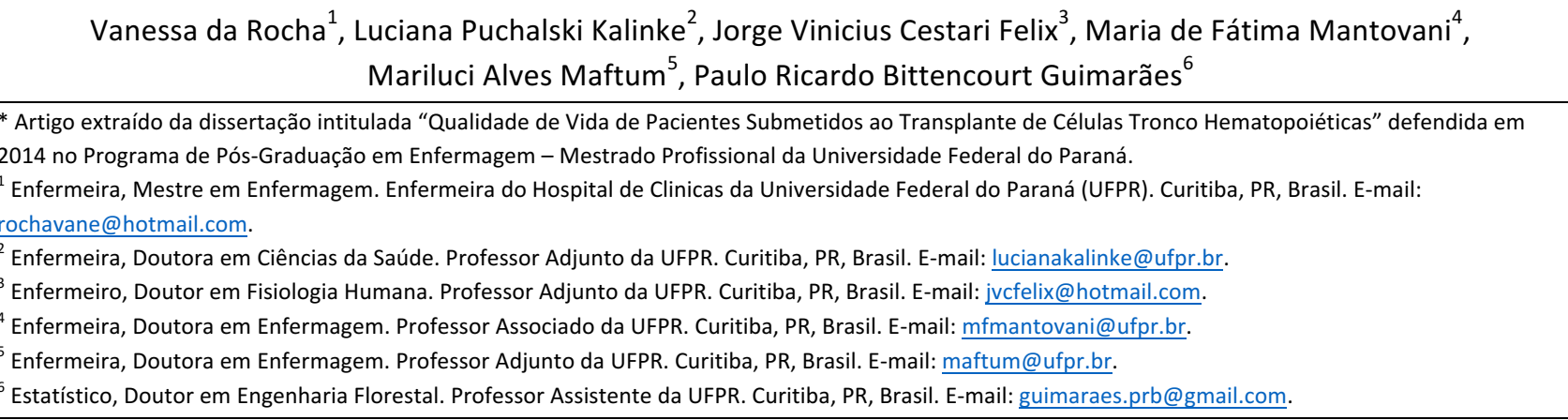

\section{RESUMO}

O objetivo deste estudo foi avaliar a qualidade de vida e identificar os domínios alterados de pacientes adultos, com câncer hematológico submetidos ao transplante de células tronco hematopoiéticas durante o período de hospitalização. Estudo longitudinal, observacional e analítico, realizado em um hospital de referência em transplante de células tronco hematopoiéticas. A coleta de dados ocorreu entre setembro de 2013 a setembro de 2014 incluiu 25 pacientes com o uso dos questionários de caracterização sociodemográfica e clínica, QLQ-C30 e FACT-BMT. Demonstraram piora estatisticamente significativa $(p<0,05)$ na qualidade de vida global, escalas funcionais, função física, social e familiar, desempenho pessoal, preocupações adicionais e sintomas, fadiga, náusea e vômito, dor, perda de apetite e diarreia. Há comprometimento da qualidade de vida dos participantes durante o período de hospitalização para o transplante. Este estudo poderá subsidiar o direcionamento das ações para o suporte adequado em todo o percurso terapêutico.

Descritores: Qualidade de Vida; Transplante de Células-Tronco Hematopoéticas; Neoplasias Hematológicas; Enfermagem Oncológica.

\section{ABSTRACT}

The objective of this study was to assess the quality of life and to identify the altered domains of adult patients with blood cancer, submitted to hematopoietic stem cells transplantation during hospitalization time. A longitudinal, observation and analytical study, conducted in a reference hospital for hematopoietic stem cell transplant. The data collection was during September of 2013 and September of 2014, including 25 patients and using questionnaires for sociodemographic and clinic characteristics, QLQ-C30 and FACT-BMT. A significant statistical worsening $(p<0.05)$ was found in global quality of life, functional scales, physical, social and family function, personal performance, additional worries and symptoms, fatigue, nausea and vomit, pain, loss of appetite and diarrhea. There is impairment in quality of life of patients during hospitalization for the transplantation. This study can subsidize the direction of actions for adequate support during all therapeutic period.

Descriptors: Quality of Life; Hematopoietic Stem Cell Transplantation; Hematologic Neoplasms; Oncology Nursing. 


\section{INTRODUÇÃO}

A necessidade de cuidados especiais durante a hospitalização aos pacientes submetidos a transplante de células tronco hematopoiéticas (TCTH) causam intensas alterações nos papéis sociais no contexto da família, do trabalho e da sociedade. Diante disso, a qualidade de vida (QV) pode ficar comprometida pela abrupta ruptura do cotidiano, invadido por novas rotinas no percurso terapêutico ${ }^{(1)}$.

Para 2015, foram calculados pela American Cancer Society um total de 162.020 novos casos de câncer hematológico nos Estados Unidos, sendo 80.900 casos de linfomas, 54.270 de leucemias e 26.850 de mielomas múltiplos. O índice de mortalidade para essas doenças é de $35 \%$ e, corresponde a aproximadamente $10 \%$ da mortalidade dos cânceres no geral ${ }^{(2)}$. O Instituto Nacional de Câncer destaca, para o Brasil, a incidência de dois tipos de câncer hematológico, as leucemias e os linfomas, com 21.340 novos casos em 2014/2015, com crescimento de aproximadamente $18 \%$ em relação à estimativa anterior $^{(3)}$.

Pacientes com diagnóstico de câncer hematológico têm sido beneficiados com o TCTH, entretanto enfrentam uma etapa difícil em suas vidas. No auge da fase produtiva, vivenciam o drama da doença, a complexidade do tratamento e os riscos a que estão expostos. Experimentam problemas físicos, emocionais e psicológicos, preocupações financeiras e com familiares, frustrações, isolamento social que comprometem sua QV. Isto exige mudanças nas atividades diárias, nos estudos, no trabalho, nas atividades de lazer e no convívio familiar e social $^{(1,4)}$.

Diante das questões abordadas acima, é necessário empreender melhorias nas modalidades de assistência de Enfermagem ao paciente submetido à TCTH em todas as etapas do prolongado tratamento ${ }^{(5)}$. Estudos que avaliam a QV em pacientes com câncer ${ }^{(6-10)}$ têm auxiliado os profissionais da saúde na identificação do comprometimento dos domínios físico, emocional, social, pessoal e familiar, bem como o impacto das intervenções pelas quais o paciente foi submetido ${ }^{(11)}$.
Assim, o objetivo deste estudo foi avaliar a QV e identificar os domínios alterados de pacientes adultos, com câncer hematológico submetidos ao TCTH durante o período de hospitalização.

\section{MÉTODO}

Estudo longitudinal, observacional e analítico, realizado no Serviço de Transplante de Medula Óssea (STMO) de um hospital pioneiro e de referência no Brasil para o TCTH, responsável por aproximadamente $16 \%$ dos transplantes alogênicos realizados no país ${ }^{(12)}$.

Foram incluídos no estudo 25 pacientes adultos com diagnóstico confirmado de câncer hematológico, atendidos no período de setembro de 2013 a setembro de 2014, com proposta de TCTH. Foram excluídos pacientes sem condições físicas ou neurológicas para preenchimento dos questionários

A coleta de dados obedeceu às seguintes etapas: (1) Pré-transplante; (2) Pós-transplante imediato, caracterizado pelo período de pancitopenia, (período em que a imunidade do paciente está mais comprometida e que ocorrem as principais complicações); (3) Fase da "pega" do Enxerto, (período em que o paciente se prepara para a alta hospitalar). Dos 25 participantes que realizaram a primeira etapa de coleta de dados, apenas 24 constituíram a amostra nas etapas subsequentes devido a um óbito.

Para a coleta de dados utilizou-se três instrumentos: (Q1) Dados Sociodemográficos e Clínicos; (Q2) Quality of Life Questionnarie-C30 (QLQ-C30) - versão 3.0 português - Brasil; (Q3) Functional Assessment Cancer TreatmentBone Marrow Transplantation (FACT-BMT) - versão 4.0 português - Brasil específico para TCTH.

Os instrumentos Q2 e Q3 foram traduzidos e validados por duas instituições independentes que desenvolvem pesquisas para o tratamento do câncer. Uma europeia, a European Organization Research Treatment of Cancer (EORTC), outra norte americana a Functional Assessment of Chronic IIIness Therapy (FACIT), ambas autorizaram a utilização dos questionários. 
O QLQ-C30, elaborado pela EORTC é composto por 30 itens divididos em: cinco escalas funcionais; três escalas de sintomas; seis itens individuais que avaliam sintomas; escala de avaliação do impacto financeiro, do tratamento e da doença e duas perguntas sobre o estado geral de saúde e QV. O FACT-BMT elaborado pela FACIT é composto por 50 itens divididos em cinco domínios: bemestar físico, funcional, emocional, social e familiar e preocupações adicionais.

Os dados sociodemográficos foram analisados com estatística descritiva expressos em frequência simples e absoluta. Os resultados do QLQ-C30 foram expressos em pontuações que variam de zero a 100 conforme o Scoring Manual da EORTC ${ }^{(13)}$. Um escore maior nas escalas funcionais representa melhor estado geral. Para os sintomas, um escore maior representa pior estado geral. Os resultados do FACT-BMT foram expressos em pontuações que variam de zero a 148. Para os domínios bem-estar físico, funcional, social e familiar e emocional o escore varia de zero a 28, preocupações adicionais variam de zero a 40, Trial Outcome Index (TOI) de zero a 96, Functional Assessment Cancer Treatment General (FACTG) de zero a 108, conforme o Scoring Manual da $\mathrm{FACIT}^{(14)}$. No escore final do FACT-BMT, os escores maiores representam melhor QV.

Foi realizada análise descritiva das escalas para resumo dos dados, expressas em média ( $M)$, desvio padrão (DP) e valores mínimos e máximos que cada item pode assumir. Para a comparação entre as etapas, foi aplicado o Teste de Friedman complementado pelo teste de Diferença Mínima Significativa de comparações múltiplas ( $p$ valor). Resultados com valor de $p \leq 0,05$ foram considerados significantes.

Esta pesquisa está aprovada pelo Comitê de Ética em Pesquisa do Setor de Ciências da Saúde da Universidade Federal do Paraná sob o parecer no 411.548. Os participantes foram esclarecidos sobre os objetivos e a metodologia da pesquisa, bem como foram sanadas todas as dúvidas existentes. Assim, os pacientes que aceitaram participar da pesquisa foram convidados a assinar o Termo de Consentimento Livre e Esclarecido.

\section{RESULTADOS}

Com base nos dados sociodemográficos, a média de idade dos participantes foi de 36 anos, com variação dos extremos de idade de 18 a 69 anos, 52\% (n=13) são do sexo feminino, $72 \%(n=18)$ da etnia branca, $56 \%(n=14)$ casados ou em união estável e $48 \%(n=12)$ possuem de um a três filhos. Quanto à escolaridade, 56\% ( $n=14)$ completaram o ensino médio e $52 \%(n=13)$ declararam-se economicamente ativos, com renda familiar mensal de até três salários mínimos $(\mathrm{R} \$ 724=$ salário mínimo nacional).

Em relação ao diagnóstico, $64 \% \quad(n=16)$ eram leucemias e $52 \%(n=13)$ com tempo de diagnóstico de um a três anos. Foram submetidos ao TCTH alogênico não aparentado $44 \%(n=11)$, e a fonte de células foi periférica para $64 \%(n=16)$ dos pacientes.

Os resultados dos questionários QLQ-C30 demonstram que a QV global inicial dos participantes assumiu valor médio de 83 . Na segunda e terceira etapa observou-se, respectivamente, que os valores médios da QV global tiveram queda significativa 56,25 e 69,44. Nas escalas funcionais, as médias basais variaram de 50 a 92, sendo os valores relacionados aos domínios social e cognitivo, respectivamente. As médias destes domínios nas etapas subsequentes foram de 25,69 a 85,42 e 34,72 a 95,83. O domínio social assumiu o valor médio mais baixo na segunda e terceira etapa. Nas escalas de sintomas, a insônia com média 20 e dificuldades financeiras com média 44 foram os mais frequentes no momento basal. Na pancitopenia a dor foi mais predominante, obtendo média de 59,72, com destaque para a perda de apetite, média de 58,33, que manteve média elevada de 50 até o momento pré-alta.

Quanto à comparação dos resultados dos questionários QLQ-C30 obtidos nas três etapas (Tabela 1), foi possível observar que houve piora estatisticamente significativa na qualidade de vida global, escalas funcionais e escalas de sintomas. 
Tabela 1: Escores significativos do QLQ-C30 na comparação entre as três etapas do estudo. Curitiba, PR, Brasil, 2014.

\begin{tabular}{|c|c|c|c|c|}
\hline Domínios & $p$ valor & 19 etapa X 2a etapa & 19 etapa X 3a etapa & 2a etap X 3a etapa \\
\hline Qualidade de vida global & $0,000001^{*}$ & $p<0,001^{*}$ & - & $p<0,05^{*}$ \\
\hline \multicolumn{5}{|l|}{ Escalas Funcionais } \\
\hline Função física & $0,00016 *$ & $p<0,001^{*}$ & - & - \\
\hline Desempenho pessoal & 0,00003* & $p<0,001^{*}$ & $p<0,05^{*}$ & - \\
\hline Função emocional & - & - & - & - \\
\hline Função cognitiva & - & - & - & - \\
\hline Função social & $0,00176 *$ & $p<0,05^{*}$ & - & - \\
\hline \multicolumn{5}{|l|}{ Escalas de Sintomas } \\
\hline Fadiga & 0,00003* & $p<0,001^{*}$ & $p<0,01^{*}$ & - \\
\hline Náusea e vômito & $0,00001^{*}$ & $p<0,001^{*}$ & $p<0,05^{*}$ & - \\
\hline Dor & $0,000001^{*}$ & $p<0,001^{*}$ & - & $p<0,001^{*}$ \\
\hline Dispneia & - & - & - & - \\
\hline Insônia & - & - & - & - \\
\hline Perda de apetite & $0,000001^{*}$ & $p<0,001^{*}$ & $p<0,01^{*}$ & - \\
\hline Constipação & - & - & - & - \\
\hline Diarreia & $0,000001^{*}$ & $p<0,001^{*}$ & - & $p<0,01^{*}$ \\
\hline Dificuldades financeiras & - & - & - & - \\
\hline
\end{tabular}

NOTA: *Dados estatisticamente significantes

Os resultados referentes aos questionários FACT-BMT estão descritos na Tabela 2. Quanto à comparação dos resultados dos questionários FACT-BMT obtidos nas três etapas
(Tabela 3), foi possível observar que o bem-estar emocional não teve piora estatisticamente significante, mas, todos os demais domínios demonstraram piora.

Tabela 2: Resultados FACT-BMT obtidos nas três etapas do estudo. Curitiba, PR, Brasil, 2014.

\begin{tabular}{cccccccccccccc}
\hline FACT-BMT & \multicolumn{3}{c}{ Basa $(\mathbf{n = 2 5})$} & & \multicolumn{3}{c}{ Pancitopeni (n=24) } & \multicolumn{2}{c}{ Pré-alta hospitala (n=24) } \\
\hline Domínios & Méd & Mín & Máx & DP & Méd & Mín & Máx & DP & Méd & Mín & Máx & DP \\
\hline Bem-estar físico & 24,6 & 11 & 28 & 4,3 & 15,6 & 3 & 26 & 6,8 & 21,2 & 11 & 27 & 4,5 \\
Bem-estar social e familiar & 21,2 & 9 & 28 & 5,1 & 16,7 & 7 & 23 & 3,8 & 15,8 & 9 & 22 & 3,1 \\
Bem-estar emocional & 19,9 & 9 & 24 & 3,7 & 20,7 & 14 & 24 & 2,6 & 21,7 & 11 & 24 & 2,8 \\
Bem-estar funcional & 21,9 & 6 & 28 & 4,7 & 14,2 & 5 & 25 & 4,4 & 15,7 & 7 & 25 & 4,7 \\
Preocupações adicionais & 31,4 & 13 & 39 & 5,3 & 23,2 & 16 & 32 & 4,6 & 24,4 & 16 & 32 & 3,9 \\
TOI*1 & 78 & 30 & 94 & 12,6 & 53,1 & 30 & 80 & 13,7 & 61,4 & 40 & 80 & 10,9 \\
FACTG*2 & 87,8 & 35 & 103 & 14,5 & 67,4 & 44 & 89 & 11,3 & 74,6 & 44 & 87 & 10,5 \\
QV geral & 119,2 & 48 & 140 & 18,7 & 90,6 & 61 & 118,2 & 15 & 99,1 & 62 & 118,2 & 13,9 \\
\hline
\end{tabular}

NOTA: *1: TOI - Índice de avaliação do resultado do tratamento *2: FACTG - Avaliação geral

Tabela 3: Escores significativos do FACT-BMT na comparação entre as três etapas do estudo. Curitiba, PR, Brasil, 2014.

\begin{tabular}{|c|c|c|c|c|}
\hline Domínios & $p$ valor & 1a etapa X 2 a etapa & 19 etapa X 3a etapa & 2a etapa X 3a etapa \\
\hline Bem-estar físico & $0,000001^{*}$ & $p<0,001^{*}$ & - & $p<0,05^{*}$ \\
\hline Bem-estar social e familiar & $0,000001^{*}$ & $p<0,01^{*}$ & $p<0,001^{*}$ & - \\
\hline Bem-estar emocional & - & - & - & - \\
\hline Bem-estar funcional & $0,000001^{*}$ & $p<0,001^{*}$ & $p<0,001^{*}$ & - \\
\hline Preocupações adicionais & $0,000001^{*}$ & $p<0,001^{*}$ & $p<0,001^{*}$ & - \\
\hline TOI & $0,000001^{*}$ & $p<0,001^{*}$ & $p<0,001^{*}$ & - \\
\hline FACTG & $0,000001^{*}$ & $p<0,001^{*}$ & $p<0,01^{*}$ & - \\
\hline QV geral & $0,000001^{*}$ & $p<0,001^{*}$ & $p<0,001^{*}$ & - \\
\hline
\end{tabular}

*Dados estatisticamente significantes

\section{DISCUSSÃO}

Neste estudo, os pacientes com cânceres hematológicos submetidos à TCTH, são casados ou estão em união estável e tiveram de um a três filhos, resultados corroborados com pesquisas internacionais ${ }^{(7-8)}$. Tal fato remete a equipe multidisciplinar a necessidade de planejamento assistencial que insira os familiares/acompanhantes em suas orientações, para auxiliar o paciente no enfrentamento das alterações físicas, psicológicas e sociais provenientes da terapêutica. 
O nível médio de escolaridade diverge de pesquisas realizadas em Chicago (EUA), que comparou a QV de pacientes submetidos ao TCTH, e de pesquisa em Omaha (EUA), que avaliou a QV e a intensidade de sintomas no TCTH, em ambas há prevalência de pacientes com ensino superior. Este estudo corrobora com estas mesmas pesquisas em relação aos dados econômicos, os participantes eram economicamente ativos, antes da hospitalização e no momento do TCTH encontravam-se afastados de suas atividades profissionais, em licença para tratamento de saúde ${ }^{(7,15)}$.

Entre os vários eventos negativos do câncer, a dificuldade financeira é responsável por condições de vulnerabilidade. A falta de recursos financeiros é um problema familiar, quando a doença afeta o membro provedor de sustento, levando-o ao afastamento do trabalho. $O$ trabalhador que adoece passa a ter um ganho menor, em forma de aposentadoria ou auxílio-doença, e não tendo outra fonte de renda, a família sofre a falta de dinheiro. Mesmo com o tratamento assumido pelo SUS, não há cobertura para as despesas com remédios receitados.

A compreensão das influências dos problemas financeiros, que se acentuam com os gastos gerados pelo tratamento, torna-se importante para que a enfermagem exerça a função de orientação, promovendo esclarecimentos sobre os direitos do indivíduo com câncer, sugerindo opções menos onerosas quando necessária alguma aquisição ao transplantado, e encaminhando os familiares aos profissionais de Serviço Social.

Em relação aos dados clínicos, as leucemias, são os cânceres hematológicos mais prevalentes seguidas pelo linfoma e mieloma ${ }^{(7)}$. O tempo de diagnóstico destes é de um a três anos (52\%) semelhante a pesquisa de Los Angeles (EUA) ${ }^{(16)}$. Entre os participantes, $44 \%$ foram submetidos ao TCTH alogênico, dado compatível com as pesquisas desenvolvidas na Alemanha e EUA ${ }^{(6,7)}$. Quanto a fonte de células, predominou as células periféricas. Pesquisadores americanos e chineses destacam o uso de células periféricas em comparação a fonte de células da medula óssea, revelando que apesar da medula óssea apresentar menor risco de doença do enxerto contra o hospedeiro (DECH), a recuperação hematológica é mais lenta, interferindo na "pega" do enxerto da medula ${ }^{(9,17)}$.

Cada paciente terá percepções diferentes conforme sua patologia, tempo de diagnóstico e sua perspectiva de vida. Identificar os domínios que alteram no paciente com câncer hematológico que se submete ao TCTH se torna importante, visto que essas alterações refletem diretamente na sua QV.

De acordo com o Scoring Manual da EORTC ${ }^{(13)}$, a QV é um construto amplo, subjetivo e, o estado geral de saúde encontrado no QLQ-C30 ou no FACT-BMT deve ser utilizado como uma medida de síntese global da QV dos pacientes oncológicos submetidos ao $\mathrm{TCTH}^{(14)}$. Os pacientes antes de iniciar o TCTH consideram sua QV boa. Entretanto, quando ocorre o período de pancitopenia, há uma queda estatisticamente significante neste índice, o que demonstra o quão severo é o tratamento.

Nas revisões publicadas no ano de 2012, que resumem os dados disponíveis sobre os escores QLQ-C30 a partir de estudos publicados sobre $\mathrm{TCTH}^{(6)}$ e com FACT$\mathrm{BMT}^{(7)}$, foi observado menor variação dos valores de mínimo e máximo e valores médios do estado geral de saúde durante hospitalização e pré-TCTH e o valor médio no momento da alta. As variações se mostram equivalentes ao presente estudo, fato que indica QV prejudicada durante o internamento, com melhora gradual no prosseguimento.

Analisando as escalas funcionais do QLQ-C30 e FACTBMT, foi observado déficit na função física/bem-estar físico e no desempenho pessoal/bem-estar funcional ao longo da terapêutica, com diferenças estatisticamente significantes entre as etapas do estudo. Esses dados corroboram com pesquisa realizada com uso da escala QLQ-C30 em 40 indivíduos que receberam intervenção e 40 indivíduos do grupo controle, com cânceres hematológicos submetidos ao TCTH alogênico. No grupo controle, semelhante aos resultados deste estudo, a 
função física no período basal foi 76, com valores menores nas etapas de hospitalização, assim como 30 a 40 dias pós-TCTH (equivalente ao período de alta), com valores médios de 53,5 e 63,5, respectivamente aos períodos $^{(10)}$. Estes resultados denotam comprometimento da QV dos pacientes do atual estudo em relação a esses domínios.

Nos dados da pesquisa de Chicago (EUA), na escala FACT-BMT, a média encontrada para o bem-estar físico variou nos três períodos entre 17,73 e 21,73, designando impacto negativo na QV, com destaque para o período de internamento, como ocorreu neste estudo, em que o valor médio durante a hospitalização foi $15,67^{(7)}$.

O domínio bem-estar funcional apresenta resultados significativos, predominantes nas avaliações de QV, ele interfere em outros sintomas relacionados à terapêutica como, fadiga, fraqueza física, perturbação do sono e falta de apetite ${ }^{(15)}$. No presente estudo, o bem-estar funcional e desempenho pessoal apresentaram piora significativa no transcorrer do tratamento, com destaque para o período de pancitopenia. Neste período até os primeiros 100 dias o bem-estar funcional permanece com piores escores, retornando aos valores basais ao completarem um ano pós-TCTH, seguindo em melhoria contínua ${ }^{(18)}$. As principais dificuldades vivenciadas são no momento de alta hospitalar, quando o paciente se encontra debilitado para exercer suas atividades laborais e diárias em seu domicílio $^{(19)}$.

No período em que o paciente permanece internado, destacam-se os inúmeros efeitos colaterais advindos do regime de condicionamento, assim a função física e o desempenho pessoal são domínios que estarão bastante comprometidos neste momento. Ao estar hospitalizado em isolamento, esse indivíduo requer cuidados que devem ser ampliados para além do modelo biomédico, atentando para outras demandas que poderão surgir no curso do tratamento, que devem ser orientadas pelo enfermeiro. Esses cuidados devem englobar medidas facilitadoras que adaptem o paciente à realidade vivenciada, estimulando sua autonomia frente às adversidades do câncer.

No presente estudo, a função social da escala QLQC30 teve média basal: 50, pancitopenia: 25,69 e pré-alta: 34,72 . Resultado semelhante à pesquisa alemã com valor médio de 53,8 na etapa basal, nas etapas de hospitalização assim como no período de alta, a mesma função social manteve valor médio 42,1 e $46,7^{(10)}$. A pesquisa que utilizou a escala FACT-BMT, revelou valor basal no bem-estar social e familiar de 24,35, pouco acima do encontrado neste estudo $(21,29)$, porém as demais etapas avaliadas não são compatíveis com este estudo ${ }^{(8)}$.

Variáveis como sexo e idade podem estar associadas ao bem-estar social, e pode ser comprometido nos pacientes que apresentam $\mathrm{DECH}^{(16)}$. Assim, pode-se inferir que complicações clínicas acarretam em prejuízos à QV do paciente submetido ao TCTH, inclusive em relação ao ajustamento psicossocial. O apoio social pode servir como um "amortecedor" levando ao melhor enfrentamento, melhora do humor e, consequentemente, melhores resultados de saúde ${ }^{(20-21)}$. Ademais, a construção de apoio social pode incorporar elementos de que tem sido associados com melhora da função imune levando a resultados clínicos positivos ${ }^{(22)}$.

Percebe-se uma limitação em discorrer sobre o bemestar social e familiar devido à carência de literaturas que investiguem a QV enquanto o paciente permanece hospitalizado para o TCTH, tendo em vista que esse é o período que pode evidenciar o comprometimento desse domínio. Destarte é importante que a enfermagem se aproprie de cuidados que se estendam a todas as dimensões comprometidas da QV, estabelecer uma relação de apoio e confiança com pacientes e familiares/acompanhantes é uma ação fundamental para este processo.

Analisando as escalas de sintomas do QLQ-C30, há aumento da sintomatologia referente à fadiga, náusea e vômito, dor, perda de apetite e diarreia ao longo da terapêutica. No FACT-BMT a perda de apetite foi o sintoma mais predominante nas etapas do presente 
estudo. Achados semelhantes foram encontrados por pesquisa realizada em Omaha (EUA), o sintoma perda de apetite assume valores médios elevados desde o período de condicionamento, com pico no período de pancitopenia, e com leve regressão após enxerto ${ }^{(15)}$, os efeitos fisiológicos acarretam piora na QV, entretanto são os domínios que a equipe multidisciplinar está mais preparada para atuar.

Quanto às preocupações adicionais com dados referentes à imagem corporal, sexualidade e dificuldades financeiras, nos resultados do FACT-BMT denotou comprometimento da QV dos participantes em relação a este domínio. Corroborando com a pesquisa de Chicago $(E \cup A)^{(7)}$, o valor de $p$ foi similar para as mesmas etapas. A terapêutica instituída pode afetar diversas dimensões da vida dessas pessoas, estando diretamente relacionada à sintomatologia e à percepção da imagem corporal pelos pacientes que se submetem ao TCTH.

Alterações da imagem corporal foram relatadas em alguns pacientes após o tratamento do transplante, incluindo a perda de cabelo e de massa muscular, erupções na pele, sensibilidade da pele ou secura, cicatrizes, alterações de peso e edema, especialmente do rosto. A imagem corporal alterada pode perturbar sentimentos sobre si mesmo como um ser sexual ${ }^{(23)}$.

Item que diminuiu significativamente do período basal até o momento de alta, no domínio das preocupações adicionais, foi em relação ao interesse pela atividade sexual $^{(7)}$. Doses elevadas de radiação e quimioterapia antineoplásica estão associadas a numerosos efeitos tóxicos a curto e longo prazo, o que pode resultar em alterações no funcionamento e satisfação sexual. Embora, o impacto de uma doença com risco de vida sobre a sexualidade talvez não seja a primeira ou mais imediata preocupação para pacientes submetidos a transplante, é um aspecto importante e por vezes esquecido de recuperação e QV após o tratamento $^{(23)}$.

Um dos principais determinantes da disfunção sexual em pacientes submetidos ao TCTH alogênico é a DECH, que pode se manifestar em diversos órgãos, ocasionando aderências nos vasos sanguíneos, erupções cutâneas e aumento da sensibilidade da pele ao redor do pênis. Nos órgãos genitais femininos pode levar à estenose vaginal $^{(24)}$.

Uma das funções inerentes ao enfermeiro é cuidar de forma integral e humanizada, fornecendo informações pertinentes aos pacientes, bem como aos seus companheiros, a fim de esclarecer sobre alterações na imagem corporal e sexualidade durante e após o tratamento, as possíveis alterações relacionadas às medicações e/ou às modalidades terapêuticas que podem afetar o desempenho sexual para que possam adequar-se a essas mudanças da melhor forma possível.

As dificuldades financeiras pelo questionário QLQC30, não houve diferença significativa entre as etapas, porém foi um dos itens que manteve valores médios, entre 37,5 e 44 . Neste estudo, $56 \%$ vivem com auxílio do governo, ou seja, são pessoas que estão incapacitadas de realizar suas atividades laborais, se preocupam com a questão financeira e normalmente são provedores da família.

\section{CONCLUSÃO}

Os resultados deste estudo demonstram que o TCTH altera a QV dos pacientes com cânceres hematológicos durante o internamento. Estas alterações foram percebidas na QV global/geral, função física/bem-estar físico, desempenho pessoal/bem-estar funcional, função social/bem-estar social e familiar, preocupações adicionais, além dos sintomas fadiga, náusea e vômito, dor, perda de apetite e diarreia.

A partir do encontrado foi possível refletir sobre o cuidado realizado atualmente pela enfermagem, no qual a sintomatologia relacionada ao tratamento é o foco principal das orientações realizadas. Frente a isso, o enfermeiro deve se fazer presente em cada momento do percurso terapêutico, promovendo ações que busquem o equilíbrio entre a manutenção das dimensões físicas, emocionais e sociais, considerando em seus cuidados e 
orientações as características pessoais e sociais de cada paciente, visando adaptá-lo ao momento vivenciado com melhores condições de vida.

É possível destacar que as associações estatísticas observadas neste estudo entre as variáveis dos questionários de QV reproduzem resultados similares na literatura, porém, consideram-se como limitadores as etapas que as pesquisas abordam, pois nem todas foram semelhantes a este estudo, algumas pesquisas não avaliam a QV no período de pancitopenia, apenas o basal e 30 dias, ou basal e pega do enxerto.

Como outra limitação deste estudo, aponta-se o tamanho reduzido da amostra, por isso os resultados se aplicam apenas a essa mesma população. Assim sendo, não podemos generalizar os resultados obtidos. Acresce ainda que este estudo trabalhou apenas com a etapa de hospitalização do TCTH. Diante disto, futuras pesquisas são importantes para que abordem outras etapas de TCTH.

\section{AGRADECIMENTOS:}

Agradecemos a European Organization Research Treatment of Cancer e a Functional Assessment of Chronic Illness Therapy que autorizaram a utilização dos questionários.

from randomized controlled trials. Ann Hematol. 2012; 91(3):427-437.

10. Wiskemann J, Dreger P, Schwerdtfeger R, Bondong A, Huber $\mathrm{G}$, Kleindienst $\mathrm{N}$, et al. Effects of a partly selfadministered exercise program before, during, and after allogeneic stem cell transplantation. Blood. 2011; 117(9):26042613.

11. Gujral S, Avery KN, Blazeby JM. Quality of life after surgery for colorectal cancer: clinical implications of results from randomised trials. Support Care Cancer. 2008; 16(2):127-132. 12. Hospital de Clínicas da Universidade Federal do Paraná, Unidade de Hematologia, Hemoterapia e Oncologia. 2013. Disponível em: http://www.hc.ufpr.br/?q=node/38. Acesso em: 09 Dezembro 2014.

13. Fayers PM, Aaronson NK, Bjordal K, Groenvold M, Curran D, Bottomley A. EORTC Quality of Life Group. 2001. The EORTC QLQ-C30 scoring manual. Disponível em:

http://www.eortc.be/qol/files/SCManualQLQ-C30.pdf. Acesso em: 05 Março 2013.

14. Eremenco SL, Cella D, Arnold BJ. A comprehensive method for the translation and cross-cultural validation of health status questionnaires. Eval Health Prof. 2005; 28(2):212-232.

15. Cohen MZ, Rozmus CL, Mendoza TR, Padhye NS, Neumann J, Gning I, et al. Symptoms and quality of life in diverse patients undergoing hematopoietic stem cell transplantation. J Pain Symptom Manage. 2012; 44(2):168-180.

16. Grant M, Cooke L, Williams AC, Bhatia S, Popplewell L, Uman $\mathrm{G}$, et al. Functional status and health-related quality of life among allogeneic transplant patients at hospital discharge: a comparison of sociodemographic, disease, and treatment characteristics. Support Care Cancer. 2012; 20(11):2697-2704. 17. Körbling M, Freireich EJ. Twenty-five years of peripheral blood stem cell transplantation. Blood. 2011; 117(24):64116416. 
18. Bevans M. Health-related quality of life following allogeneic hematopoietic stem cell transplantation. Hematol. 2010;

1:248-254.

19. Pidala J, Anasetti C, Jim H. Quality of life after allogeneic hematopoietic cell transplantation. Blood. 2009; 114(1):7-19.

20. Foster LW, McLellan L, Rybicki L, Dabney J, Copelan E,

Bolwell B. Validating the positive impact of in-hospital lay carepartner support on patient survival in allogeneic BMT: a prospective study. Bone Marrow Transplant. 2013; 48(5):671677.

21. Beattie S, Lebel S, Tay S. The influence of social support on hematopoietic stem cell transplantation survival: a systematic review of literature. Plos One. 2013; 8(4):1-5.

22. Lutgendorf SK, Sood AK. Biobehavioral factors and cancer progression: physiological pathways and mechanisms.

Psychosom Med. 2011; 73(9):724-730.

23. Thygesen $\mathrm{KH}$, Schjodt I, Jarden M. The impact of hematopoietic stem cell transplantation on sexuality: a systematic review of the literature. Bone Marrow Transplant. 2012; 47(5): 716-724.

24. Wong FL, Francisco L, Togawa K, Kim H, Bosworth A, Atencio $L$, et al. Longitudinal trajectory of sexual functioning after hematopoietic cell transplantation: impact of chronic graft-versus-host disease and total body irradiation. Blood. 2013; 122(24):3973-3981.

Recebido: 10/06/2015.

Aceito: 25/09/2015.

Publicado: 31/12/2015. 